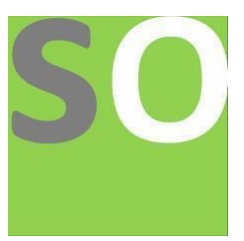

Article title: I MODI DI ASSUNZIONE DEL DEBITO ALTRUI: DELEGAZIONE, ESPROMISSIONE, ACCOLLO. Authors: Raffaele Ambrosino[1]

Affiliations: civil lawyer in Italy, Foggia, 71121, Via molfetta 15[1]

Orcid ids: 0000-0003-2365-1140[1]

Contact e-mail: raffaele.ambrosino.1992@gmail.com

License information: This work has been published open access under Creative Commons Attribution License $\mathrm{http}: / / c r e a t i v e c o m m o n s . o r g / l i c e n s e s / b y / 4.0 /$, which permits unrestricted use, distribution, and reproduction in any medium, provided the original work is properly cited. Conditions, terms of use and publishing policy can be found at https://www.scienceopen.com/.

Preprint statement: This article is a preprint and has not been peer-reviewed, under consideration and submitted to ScienceOpen Preprints for open peer review.

DOI: 10.14293/S2199-1006.1.SOR-.PPVJGW7.v1

Preprint first posted online: 14 September 2021

Keywords: obbligazioni, diritto civile, debiti, responsabilità civile, crediti circolazione del credito, creditore, debitore. 


\section{MODI DI ASSUNZIONE DEL DEBITO ALTRUI: DELEGAZIONE, ESPROMISSIONE, ACCOLLO.}

\section{Considerazioni preliminari}

Delegazione, espromissione ed accollo sono istituti giuridici tipizzati che trovano espressa disciplina nel codice civile e la cui applicazione determina, in vario modo, l'assunzione del debito altrui.

Vista la capacità di tali negozi di modificare il lato passivo del rapporto obbligatorio, è essenziale definire preliminarmente la differenziazione giuridica tra due fenomeni cui si ricollega la modificazione ex latere debitoris: la successione nel debito e la novazione soggettiva in generale. Dal punto di vista giuridico la successione nel lato passivo dell'obbligazione si concretizza in un atto di subingresso di un terzo nella posizione del debitore originario, il che comporta una totale sostituzione del successore che surroga il debitore nella totalità dei poteri e dei doveri connessi al rapporto obbligatorio. Presupposto della successione nel debito è dunque il necessario consenso del creditore della prestazione e conseguenza del consenso legittimamente prestato è la liberazione del soggetto passivo da ogni tipo di pretesa concernente il rapporto obbligatorio. Da quanto precisato quindi può affermarsi pacificamente che il fenomeno successorio non costituisce un'idonea categoria giuridica per inquadrare gli istituti della delegazione, espromissione ed accollo, i quali, come si vedrà, non determinano né una piena liberazione del debitore originario né un subingresso del nuovo debitore nella medesima posizione giuridica del soggetto passivo originario con la relativa acquisizione della titolarità dei poteri di eccezione esperibili contro il creditore.

Ancor più discussa è la riconducibilità degli istituti de quibus alla categoria della novazione soggettiva in quanto ad essere oggetto di discussione è la stessa valenza del concetto di novazione per la modificazione soggettiva del rapporto obbligatorio. Quando infatti si verifica la sostituzione di una delle parti dell'obbligazione, dal punto di vista giuridico non si ottiene un effetto estintivo proprio della novazione in quanto il rapporto obbligatorio rimane in vita in tutti i suoi elementi essenziali eccetto il soggetto tenuto all'adempimento.

A sostegno di tale impostazione sovviene la lettera dell' art. 1235 cod. civ. rubricato "novazione soggettiva" che sancisce la necessità di ricorrere alla disciplina degli istituti della delegazione, espromissione ed accollo (liberatori) per regolamentare i casi di sostituzione del debitore dell'obbligazione.

Considerata pertanto centrale nella dinamica del rapporto obbligatorio la posizione del soggetto debitore può concludersi che i negozi di assunzione del debito altrui non sono in grado (se non per effetto di un consenso "liberatorio") di determinare un fenomeno successorio agendo piuttosto sul piano della responsabilità debitoria "allargandola" e consentendo al creditore di fruire di un più elevato grado di probabilità di adempimento del credito.

\section{DELEGAZIONE}

\section{Nozione e natura giuridica}

Ai sensi degli artt. 1268 e 1269 cod. civ. la delegazione consiste in un'operazione giuridica mediante la quale un soggetto che prende il nome di delegante incarica un altro soggetto detto delegato o di assumere o di pagare al terzo delegatario il debito vantato da quest' ultimo nei confronti del delegante.

Dall'analisi dell'istituto emerge la necessaria presenza di tre soggetti tra i quali intercorrono diversi rapporti: in primo luogo vi è un rapporto obbligatorio principale detto di valuta 
intercorrente tra delegante e delegatario, che vede il primo debitore del secondo, ed in secondo luogo vi è un rapporto, tra delegante e delegato, detto di provvista caratterizzato solitamente dalla sussistenza di un credito da parte del primo nei confronti del secondo.

Come ravvisabile nella lettera delle disposizioni richiamate l'incarico può avere ad oggetto sia l'assunzione del debito da parte del delegato verso il delegatario, che comporta l'estensione del lato passivo del rapporto obbligatorio di valuta con l'aggiunta di un nuovo debitore, sia il pagamento della prestazione da parte dell'incaricato che determina l'adempimento del rapporto di valuta; nel primo caso si discorre di delegatio promittendi mentre nel secondo di delegatio pagamenti.

Per condurre un'analisi esaustiva delle varie teorie sorte sulla natura giuridica della delegazione è bene mettere in luce la causa dell'intera operazione che si identifica nella concentrazione delle prestazioni caratterizzanti i vari rapporti in un'unica prestazione in grado di estinguere con un unico adempimento più rapporti giuridici, il tutto in ossequio al principio di economia dei mezzi giuridici.

Relativamente alla natura giuridica dell'istituto è possibile ravvisare in dottrina l'esistenza di due teorie.

Secondo una prima tesi detta "unitaria" la delegazione si identifica in un unico negozio giuridico caratterizzato da una pluralità di parti (cd. "negozio trilatero") il quale sarebbe caratterizzato da una formazione progressiva dell'unica volontà negoziale frutto delle singole manifestazioni del consenso liberamente espresso dai soggetti coinvolti. Pertanto il rapporto causale caratterizzante ogni singolo elemento della fattispecie resta al di fuori del negozio che secondo la teoria esposta è caratterizzato da un'unica causa negoziale tipica.

A parere di altri studiosi, autori della tesi "atomistica", la delegazione è il risultato di un collegamento negoziale tra singole manifestazioni di volontà che non si fondono in un unico negozio ma sono solo teleologicamente orientate al raggiungimento di uno scopo finale comune. I singoli atti posti in essere dai tre soggetti conservano pertanto la loro autonomia causale che è in grado di riflettersi sull'intera operazione; tale tesi trova fondamento nella lettera dell'articolo 1270 cod. civ. che disciplina il diritto di revoca dell'incarico da parte del delegante ed altresì il potere del delegato di assumere l'obbligazione od effettuare il pagamento anche dopo la morte del delegante o nel caso di incapacità sopravvenuta del medesimo.

L' impostazione atomistica comporta l'obbligo di inquadrare la delegazione partendo dalla qualificazione giuridica dei singoli atti negoziali che la compongono.

In primo luogo va determinata la natura dell'atto di delega del delegante al delegato che è stata variamente classificata: ora in atto unilaterale (cd. iussum) inteso come espressione di un diritto potestativo del debitore nei confronti del delegato in grado di produrre i suoi effetti in virtù della posizione di soggezione di quest'ultimo alla luce del rapporto di provvista; ora in un atto di autorizzazione a mezzo del quale il delegante autorizza il delegato a compiere un atto di disposizione di un proprio diritto (diritto di credito oggetto del rapporto di provvista); ora in un contratto di mandato.

A differenza delle prime due impostazioni di stampo "unilaterale" la sussunzione della delega in una fattispecie contrattuale di natura gestoria trova riscontro nella lettera della norma che richiede, per il perfezionamento dell'atto, il consenso del delegato ancorché sia debitore del delegatario e quindi sottoposto ad una posizione di soggezione. In tal caso infatti con l'accettazione dell'incarico sorge una nuova fonte di obbligazione per il delegato che trova nel rapporto di provvista solo una giustificazione causale tipica di un mandato in rem propriam. 
La tesi contrattualistica appare quindi più congeniale al dettato normativo, tuttavia non va trascurato che le impostazioni unilateralistiche riacquistino il loro vigore in funzione della concreta situazione di fatto che sottende l'operazione delegatoria, a conferma di ciò sovviene la lettera della legge secondo cui la delega non può essere rifiutata, e quindi produce in modo automatico ed unilaterale i propri effetti, qualora il rapporto delegato-delegante sia regolamentato da usi diversi (si faccia l'ipotesi della delega di pagamento di un modello F24 che viene inviata dal debitore d'imposta all'istituto di credito che non può essere rifiutata da quest'ultimo qualora sul conto corrente del cliente vi siano le risorse disponibili).

L'atto di autorizzazione tra debitore principale e creditore con cui il primo assegna al delegatario un nuovo debitore si concretizza in un negozio giuridico unilaterale che spiega i propri effetti in modo recettizio, in quanto non costituisce un atto pregiudizievole per il creditore che addirittura può avvantaggiarsi di un'estensione della responsabilità per l'adempimento al patrimonio di un altro soggetto. Ovviamente la ratio dell'unilateralità degli effetti dell'atto di autorizzazione è rinvenibile nel principio che sottende la possibilità di un adempimento del terzo purché l'esecuzione della prestazione da parte di un soggetto diverso dal debitore sia esatta.

Ultimo atto negoziale dell'operazione delegatoria è il negozio di assunzione del debito da parte del delegato verso il delegatario. Tale atto giuridico è stato diversamente inquadrato dagli studiosi: per alcuni l'assunzione del debito da parte del delegato costituirebbe una vera e propria promessa unilaterale di pagamento, che sarebbe tipizzata dallo stesso articolo 1268 cod. civ. nel pieno rispetto del principio di tipicità ex art. 1987 cod. civ. ; per altra dottrina invece anche tale negozio avrebbe natura contrattuale ed il suo perfezionamento sarebbe ancorato al consenso del creditore delegatario, quest'ultimo infatti non trarrebbe un mero vantaggio dalla delegazione in quanto potrebbe potenzialmente soccombere al delegato nel caso in cui fosse suo debitore potendo il terzo assuntore del debito altrui far valere le proprie eccezioni personali ex art. 1271 co. 1 cod. civ.

\section{Disciplina}

La delegazione, così come disciplinata dal codice può assumere diverse "forme" assolvendo diverse funzioni.

In primo luogo sul piano dell'adempimento la delegazione può comportare sia l'assunzione dell'obbligo di pagamento del delegato che si aggiunge al debitore originario sia l'estinzione dell'obbligazione con il pagamento fatto dal terzo.

La prima fattispecie è la cd. delegazione di debito disciplinata dall'art. 1268 cod. civ. essa realizza una modificazione del lato passivo del rapporto obbligatorio estendendo la platea dei soggetti responsabili dell'adempimento, senza avere un immediato carattere satisfattivo.

All'interno di tale fattispecie può inoltre verificarsi un'ulteriore diversificazione dell'operazione delegatoria, infatti in base ad un potere discrezionale del creditore-delegatario, può verificarsi o una mera aggiunta del nuovo debitore al vecchio o addirittura una sostituzione del nuovo debitore con liberazione del primo. Nel primo caso la delegazione è detta cumulativa, nel secondo liberatoria.

La delegazione cumulativa costituisce la naturale evoluzione dell'operazione delegatoria, pertanto ha causa nell'assunzione del debito altrui e produce l'effetto di cumulare al soggetto passivo originario un altro debitore. Tale effetto ha delle importanti ripercussioni sul regime di 
responsabilità del debitore, l'obbligazione si trasforma in un rapporto pluripersonale dal lato passivo dando vita ad un'obbligazione solidale che modifica l'ordine di adempimento dei coobbligati.

L'art. 1268 cod. civ. stabilisce infatti che all'esito dell'operazione il delegato diventerà un debitore obbligato in solido ed in via principale rispetto all'originario debitore il quale si avvantaggerà del beneficium ordinis sancito dalla norma che vieta al creditore di chiedere l'adempimento al debitore originario prima di averlo chiesto al delegato. Ovviamente tale privilegio è confinato al piano dell'adempimento e non si estende al momento escussorio, non vi è pertanto nella norma un fondamento giustificativo di un eventuale beneficium excussionis. Alla luce di tale effetto giuridico può asserirsi che la modificazione soggettiva del rapporto obbligatorio non è solo quantitativa, ma anche qualitativa poiché produce una degradazione di responsabilità del vecchio debitore rendendo l'obbligazione sussidiaria.

Tale conseguenza è tra l'altro indice di un trasferimento del peso economico della responsabilità tra i due debitori che è sopportato dal nuovo debitore.

Queste ripercussioni trovano giustificazione nel fondamento giuridico dell'operazione in quanto il soggetto delegato bilancia il peso dell'obbligazione che ha volontariamente assunto verso il delegatario con la liberazione dal proprio debito verso il delegante "alleggerendo" con un unico adempimento il proprio status patrimoniale.

Il creditore ha però la facoltà di liberare l'originario debitore a seguito dell'assunzione del debito da parte del delegato, in tale ipotesi l'effetto liberatorio è prodotto da una espressa manifestazione di volontà del delegatario che scioglie l'originario debitore dal vincolo obbligatorio determinando in tal caso una vera e propria sostituzione del soggetto passivo del rapporto che, per quanto detto, non può ritenersi un fenomeno successorio in senso tecnico.

Dall'analisi del complesso normativo inoltre emerge che la liberazione non è "piena" infatti il delegante rimane responsabile dell'adempimento qualora il nuovo debitore sia insolvente. Questo stato di "quiescenza" del rapporto originario assume una diversa disciplina a seconda del momento in cui si manifesta l'insolvenza del delegato: se essa era preesistente all'assunzione del debito allora il fondamento causale della liberazione viene presuntamente meno, se invece l'insolvenza sopravviene al momento liberatorio allora nessun tipo di responsabilità può essere ascritta al debitore antico, se non per espressa riserva manifestata dal creditore all'atto di liberazione. Ovviamente in tal caso la responsabilità del delegante è a titolo di garanzia e non più a titolo di debitore, e soggiace pertanto al relativo regime legale.

Il delegante rimane, seppur liberato, debitore del delegatario in caso di insolvenza preesistente del delegato, o garante del medesimo in caso di liberazione con riserva qualora l'insolvenza del delegato sopravvenga.

Sul piano delle garanzie la liberazione del debitore ha un effetto diversificato a seconda del garante e della sorte della nuova obbligazione. In primo luogo ogni garanzia prestata da soggetti terzi si estingue in assenza di una volontà contraria dei garanti, e ciò rappresenta un indice dell'impossibilità di qualificare la sostituzione del debitore come successione. L'estinzione delle garanzie è tra l'altro definitiva in quanto il sopravvenire di una causa di nullità della delegazione con estinzione dell'assunzione del debito da parte del delegato non è opponibile ai terzi.

Anche le garanzie personali prestate dal debitore si estinguono, ma in caso di contraria volontà delle parti restano in vita quali garanzie prestate dal terzo poiché il debitore per effetto della liberazione è ormai estraneo al rapporto obbligatorio. Ovviamente in ogni caso in cui 
l'obbligazione antica rivive, anche le garanzie personali dell'originario debitore riacquistano efficacia, in quanto il rapporto riacquista efficacia ex tunc.

Altra classificazione dell'operazione delegatoria va fatta in base ai rapporti sussistenti tra $i$ tre soggetti coinvolti, a tal proposito la delegazione è detta passiva su debito (o coperta) tutte le volte in cui il delegante oltre ad essere debitore del delagatario è anche creditore del delegato, pertanto l'incarico delegatorio trova fondamento nella possibilità di concentrare i due adempimenti in un'unica prestazione soddisfacendo il principio di economia dei mezzi giuridici; quando invece non sussiste un rapporto di provvista tra debitore e delegato allora la delegazione è "scoperta" e la sua funzione non è più quella di concentrazione delle prestazioni in un solo adempimento ma astrae la causa dall'accordo che sottende l'assunzione dell'incarico da parte del delegato, che può essere donandi causa (generando una liberalità indiretta), o a titolo oneroso (originando un rapporto di mutuo). Nel caso in cui nessun rapporto di provvista venga posto a fondamento dell'assunzione del debito altrui, l'eventuale adempimento del terzo dà origine ad un indebito soggettivo con la conseguente esperibilità dell'azione di ripetizione nei confronti del delegante. È chiaro che in ossequio al principio di autonomia contrattuale è possibile impiegare lo schema delegatorio per dar vita a rapporti giuridici ex novo, non giustificati né da un rapporto di valuta né da un rapporto di provvista, in tal caso l'operazione sarebbe "atipica" e la disciplina della delegazione troverà applicazione solo per il rinvio operato dai soggetti coinvolti alle disposizioni in materia.

La disciplina legale della delegazione di debito è governata dal legame sussistente tra $\mathrm{i}$ vari soggetti dell'operazione e dall'eventuale rinvio operato dai medesimi ai rapporti di valuta e di provvista. A tal proposito può essere "pura" nel caso in cui il delegato, nell'atto di assunzione del debito verso il delegatario non ha fatto menzione del rapporto di provvista, oppure "titolata", nell'opposto caso in cui il terzo delegato nell'assumere il debito del delegante fa espresso riferimento al rapporto di provvista che lo lega al debitore originario e giustifica l'operazione. Tale distinzione ha notevoli ripercussioni sul regime dell'efficacia dell'intera operazione e sul regime delle eccezioni opponibili dalle parti.

Con riferimento al regime giuridico dell'efficacia la delegazione pura identificandosi in un negozio a causa astratta non viene meno qualora uno dei due rapporti che la sottendono cada nel nulla per effetto di un vizio di validità. Il delegato è sempre tenuto a pagare anche se il rapporto di provvista viene meno, così come non può far valere l'invalidità del rapporto di valuta per sottrarsi all'adempimento, infatti nonostante il venir meno di uno dei due rapporti, l'interesse all'adempimento resta in vita o per il delegante che può beneficiare dell' estinzione del debito principale, o per il creditore delegatario che beneficia dell'adempimento del terzo, o del delegato che adempiendo si libera dal proprio debito verso il delegante.

È chiaro che l'astrattezza non può spingersi a giustificare l'esistenza dell'assunzione del debito quando sono posti nel nulla entrambe i rapporti di base, in tal caso è la stessa legge a stabilire che il delegato può ritenersi liberato dall'esecuzione della prestazione in quanto priva in assoluto di ogni giustificazione causale, nel caso di specie un eventuale adempimento non gioverebbe a nessuna delle parti determinando solo un trasferimento ingiustificato di ricchezza dal delegato al delegatario nullo per assenza di causa.

Qualora invece la delegazione sia "titolata" anche solo parzialmente (le parti vincolano l'assunzione del debito al solo rapporto di valuta o di provvista), l'operazione negoziale subisce gli effetti dei rapporti sottostanti, pertanto il nuovo debitore risulta vincolato al pagamento solo nei limiti in cui i rapporti di valuta e di provvista siano validi ed efficaci, in questo caso la 
delegazione è caratterizzata da un nesso causale che pervade i vari atti negoziali che la compongono aggiungendo alla loro causa tipica un ulteriore elemento di validità ed efficacia. La classificazione appena esaminata determina un diverso regime delle eccezioni opponibili tra i vari soggetti del rapporto.

In primo luogo va osservato come la purezza della delegazione impedisca al delegato di eccepire al delegatario eccezioni fondate sul rapporto di provvista, anche se il delegatario è a conoscenza dei vizi dello stesso. L'unica salvezza è rappresentata dalla doppia invalidità che pone nel nulla la causa dell'intera operazione negoziale per il venir meno delle singole cause negoziali dei rapporti sottostanti.

A prescindere da ogni riferimento fatto dal delegato ai rapporti di base, sono sempre opponibili nei confronti del delegatario le eccezioni personali ovvero quelle opposizioni fondate su rapporti intercorrenti personalmente tra delegato e creditore, tale principio generale è in sintonia con lo scopo giuridico del negozio delegatorio, infatti l'istituto potrebbe permettere con un'unica prestazione l'estinzione non solo dei rapporti di valuta e di provvista, ma anche di un eventuale terzo rapporto di debito tra delegato e delegatario qualora quest'ultimo sia debitore del terzo assuntore del debito e gli venga eccepita la compensazione personale. Ovviamente al di là delle eccezioni con carattere satisfattivo (compensazione), le uniche circostanze opponibili dal delegato al delegatario sono quelle concernenti i vizi che colpiscono il negozio di assunzione del debito, come il difetto di capacità o un vizio dell'accordo.

Le eccezioni relative al rapporto di valuta ed a quello di provvista possono essere invece opposte solo se il terzo delegato ha fatto espresso rinvio ai rapporti sottostanti nel momento in cui ha concluso il negozio di assunzione del debito, tale richiamo pertanto costituisce una vera e propria condizione risolutiva del consenso manifestato e giustifica l'inadempimento determinando la risoluzione dell'assunzione del debito ex tunc, con l'effetto di determinare l'obbligo di ripetizione da parte del delegatario di quanto ottenuto in pagamento.

La doppia titolazione della delegazione ha quindi l'effetto di eliminare l'autonomia dei rapporti obbligatori che caratterizza invece la delegazione astratta.

La disciplina della delegazione è caratterizzata in modo tipico anche per le sue modalità di estinzione. È infatti l'art. 1270 cod. civ. a stabilire, in primo luogo, il potere di revoca del delegante esercitabile fin quando non si è manifestato il consenso del delegato all'assunzione del debito verso il creditore. In tal caso però è necessaria un'osservazione giuridica: aderendo infatti alla teoria che qualifica l'incarico delegatorio sub specie di mandato in rem propriam, può derivarsi che il potere di revoca può venir meno anche con la sola accettazione dell'incarico da parte del delegato in quanto in tal caso sarebbe necessario un atto di mutuo dissenso per lo scioglimento del vincolo delegatorio essendo l'incarico assunto dal debitor debitoris anche nel proprio interesse.

A contrario, secondo la lettera dell'art. 1270 cod. civ. impediscono l'estinzione del rapporto delegatorio la morte o la sopravvenuta incapacità del delegante, in tal caso infatti costituendo il negozio di incarico al delegato un contratto stipulato anche nell'interesse dell'incaricato, il venir meno di uno dei soggetti dell'operazione non determina l'estinzione di un interesse concreto all'adempimento del negozio; la norma è riferibile alla sola fattispecie di delegazione di debito in base alla quale il delegato accetta l'incarico in quanto debitore del delegante, in tal modo l'adempimento del terzo libererà lo stesso dall'obbligo di adempiere al debito di provvista verso i successori del delegante, o il suo rappresentante legale. 


\section{ESPROMISSIONE}

\section{Nozione e natura giuridica}

In base all'art. 1272 cod. civ. l'espromissione è un negozio mediante il quale un soggetto estraneo al rapporto obbligatorio, assume spontaneamente verso il creditore il debito oggetto di obbligazione.

Nella dinamica negoziale le parti coinvolte in modo diretto sono due: terzo espromittente e creditore esprommissario, la manifestazione di volontà dell'assuntore del debito è caratterizzata infatti dalla spontaneità di quest'ultimo pertanto il perfezionamento del negozio richiede il solo assenso del creditore, ed ogni eventuale rapporto tra terzo e debitore principale rimane estraneo alla sua conclusione.

Dalla struttura dell'istituto emerge che la funzione principale e determinante attribuitagli dalla legge è l'assunzione del debito altrui, che costituisce l'unica causa del negozio espromissorio; la ragione che spinge il terzo all'assunzione del debito è confinata alla sfera dei motivi e pertanto risulta essere estranea al negozio.

Relativamente alla natura giuridica del negozio espromissorio diverse sono le teorie avanzate dagli operatori del diritto.

In primo luogo è stata sostenuta la natura giuridica di promessa unilaterale in quanto l'assunzione del debito è generata dalla sola dichiarazione negoziale del terzo espromittente il quale si obbliga attraverso un negozio la cui efficacia è tipizzata dall'art. 1272 cod. civ., ovviamente in quanto promessa unilaterale l'espromissione produce i sui effetti tipici a prescindere dall'accettazione del creditore che ha il solo effetto di modificare (eventualmente) il regime di responsabilità ex latere debitoris con degradazione del debitore antico a debitore solidale sussidiario, o addirittura provocare la liberazione di quest'ultimo da ogni obbligo debitorio.

A parere di altri studiosi supportati dalla prassi giurisprudenziale prevalente, l'espromissione è un vero e proprio contratto, pertanto il suo perfezionamento si ottiene solo con il consenso espresso del creditore all'assunzione del debito da parte dell'espromissario.

Per tale teoria infatti il creditore non trae solo un beneficio dalla conclusione del negozio in quanto dal suo perfezionamento deriva un'automatica modificazione in pejus del regime di responsabilità debitoria in quanto l'antico debitore è responsabile solo in via sussidiaria. Tale mutamento del regime solutorio rappresenterebbe la controprestazione del creditore all'aggiunta della responsabilità del nuovo debitore e pertanto l'espromissione consisterebbe in un vero e proprio contratto sinallagmatico a prestazioni corrispettive.

Tra i “contrattualisti” vi è chi si spinge a dare un'ulteriore qualificazione dell'espromissione considerandola contratto a favore di terzo, ove il beneficiario è rappresentato dal debitore liberato in assoluto (espromissione liberatoria) o dal solo peso economico dell'adempimento (espromissione cumulativa). Tuttavia un'analisi comparativa dei due negozi giuridici permette di concludere che l'espromissione non è costruita come un contratto a favore del terzo né può esservi spazio per un'estensione analogica della disciplina ex art. 1411 cod. civ. infatti nell'espromissione manca l'elemento caratteristico del potere di revoca dello stipulante e la possibilità di rendere irrevocabile la stipulazione con l'accettazione del beneficiario, si consideri inoltre che il debitore espromesso non ha neppure la facoltà di rifiutare la stipulazione in suo favore anzi può addirittura esserne all'oscuro, pertanto i meri effetti favorevoli per il debitore di un contratto di espromissione non sono sufficienti per suffragare l'inquadramento giuridico prospettato. 


\section{Disciplina}

Dal dettato normativo che regola la disciplina dell'espromissione è possibile riscontrare una bipartizione del negozio in base agli effetti prodotti: l'espromissione può infatti essere cumulativa qualora abbia il solo effetto di provocare un'aggiunta dell'espromittente alla responsabilità del debitore espromesso, oppure può essere liberatoria provocando lo scioglimento del rapporto nei confronti del debitore originario.

L'espromissione cumulativa rappresenta la fattispecie naturale dell'istituto infatti con il solo accordo di creditore e terzo espromittente la conseguenza giuridica è la modificazione soggettiva del rapporto obbligatorio con la nascita di un'obbligazione solidale passiva governata dal principio di sussidiarietà.

Un'interpretazione "sistematica" del capo VI del Libro IV del codice civile permette di considerare l'effetto di degradazione della responsabilità del debitore espresso dall'art. 1268 cod. civ. co. 2 un principio generale dettato in materia di negozi di assunzione del debito altrui, pertanto con l'espromissione il creditore non può rivolgersi al debitore originario se prima non ha chiesto l'adempimento all'espromesso, anche in tal caso inoltre il debitore godrà solo di un beneficium ordinis e non di un beneficium escussionis.

La nuova obbligazione sarà solidale e sussidiaria, verificandosi uno spostamento del peso economico del debito in capo all'espromittente il quale in caso di adempimento non sarà titolare di un'azione di regresso verso il debitore espromesso, ma al massimo potrà agire in rivalsa in base al rapporto causale e personale che ha determinato l'iniziativa all'assunzione del debito.

La liberazione del debitore è invece l'epilogo dell'accordo espromissorio solo nel caso in cui vi sia un'espressa manifestazione di volontà del creditore che può essere ritenuta un atto unilaterale recettizio che non presuppone l'accettazione dell'espromesso, inoltre la liberazione costituisce la controprestazione dell'espromissario oggetto del sinallagma del contratto di espromissione.

A differenza degli altri negozi di assunzione del debito altrui con effetto liberatorio il legislatore non si è preoccupato di disciplinare l'eventuale stato di insolvenza del nuovo debitore, che non costituisce pertanto causa di riviviscenza della vecchia obbligazione o di garanzia

dell'espromesso neppure nel caso in cui preesisteva all'atto liberatorio. La causa giustificatrice di un tale effetto "pienamente" liberatorio è rintracciabile nella totale estraneità del debitore al contratto di espromissione; il creditore espromissario non può imputare al debitore le

conseguenze negative di un atto posto in essere in assenza di ogni coinvolgimento della parte beneficiaria.

A seguito dell'espromissione liberatoria si verifica ex art. 1275 cod. civ. l'estinzione delle garanzie che assistono il credito purché non vi sia il consenso espresso dei terzi garanti a mantenerle in vita, e l'estinzione permane anche nel caso di reviviscenza della vecchia obbligazione per effetto dell'invalidità del contratto di espromissione ex art. 1276 cod. civ. in tale evenienza solo le originarie garanzie prestate dal debitore antico riacquistano efficacia in quanto la retroattività degli effetti dell'invalidità del negozio espromissorio non possono pregiudicare i diritti dei terzi acquistati in buona fede.

Dalla disciplina concernente il regime delle eccezioni opponibili dal terzo espromittente al creditore è desumibile l'astrattezza parziale dell'istituto in quanto il rapporto di valuta incide in modo causale sullo sviluppo del nuovo rapporto obbligatorio, unico fondamento causale dell'espromissione, stante l'estraneità del debitore e dell'eventuale rapporto di provvista che lo 
lega al terzo assuntore, è rintracciabile nella sussistenza di un valido rapporto di debito tra espromissario ed espromesso.

Per quanto detto dalla stipulazione di un contratto di espromissione il terzo può opporre al creditore sia le eccezioni relative al personale rapporto che ha con la controparte legate ad altri rapporti giuridici, sia quelle oggettive scaturenti dal contratto concluso, ciò in ossequio al generale principio di economia dei mezzi giuridici, pertanto oltre a poter eccepire il difetto di capacità o le cause di nullità del contratto (di espromissione), l'espromittente può adempiere eccependo la compensazione di un credito scaturente da altro rapporto obbligatorio di cui il creditore è soggetto passivo, estinguendo con un unico atto più rapporti pendenti.

Le eccezioni relative al rapporto di valuta sono invece disponibili al nuovo debitore nei limiti in cui non abbiano carattere personale, tale parziale estensione è dovuta al fondamento causale dell'espromissione che è rintracciato nell'esistenza di un debito da espromettere. Ovviamente siccome il rapporto di valuta ha una rilevanza "oggettiva" rispetto all'espromissione, l'eventuale sussistenza di cause di invalidità o di inefficacia relative e di carattere personale, come il difetto di capacità dell'originario debitore o la compensabilità della prestazione con altro credito dell'espromesso non autorizzano il terzo assuntore ad esimersi dall'adempimento.

Un regime di carattere dispositivo è previsto per le eccezioni relative al rapporto di provvista tra terzo espromittente e debitore espromesso. In tale ipotesi essendo il contratto espromissorio causalmente estraneo ai motivi che spingono il terzo ad assumersi il debito, il rapporto eventualmente esistente tra terzo e debitore può rilevare solo se entra a far parte del contratto di espromissione per volontà espressa delle parti in virtù della libertà di determinare il contenuto del contratto. In questa circostanza il rapporto di provvista e le relative eccezioni costituiranno condizioni contrattuali dispositive del regime legale ordinario dell' istituto.

\section{ACCOLLO}

\section{Nozione e natura giuridica}

L'accollo è un negozio giuridico che coinvolge in modo diretto ed immediato il debitore di un rapporto obbligatorio ed un terzo il quale si assume l'obbligazione del primo, ed in modo indiretto ed eventuale il creditore che può aderire all'accordo tra terzo accollante e debitore accollato per giovarsi dell'estensione della responsabilità passiva al nuovo debitore.

Il negozio di accollo può considerarsi a causa variabile in quanto l'assunzione del debito da parte dell'accollante costituisce un effetto della stipulazione piuttosto che la vera e propria causa negoziale.

Tale osservazione è il frutto dell'inquadramento giuridico dell'istituto de quo che a causa delle sue diverse pratiche applicazioni è stato oggetto di diverse teorie.

Una prima impostazione tende ad escludere l'autonomia negoziale dell'accollo classificandolo come un patto accessorio di un diverso contratto, costituendo una modalità di esecuzione delle prestazioni in esso dedotte (modalità di pagamento, reciproca concessione, ecc...), da tale classificazione consegue la stretta dipendenza giuridica del negozio di accollo dal contratto principale pertanto ogni vizio o difetto di funzionamento di quest'ultimo si rifletterà sulla validità dell'assunzione del debito altrui.

La terminologia normativa però permette di ritenere l'accollo un negozio dotato di autonomia $\mathrm{e}$ di natura contrattuale che si perfeziona con solo con il consenso manifestatamente prestato dalle parti ed è dotato di una disciplina tipica ed indipendente dal rapporto di debito su cui incide. 
Le problematiche legate alla causa variabile di tale negozio vengono risolte sussumendo il contratto di accollo nella fattispecie di contratto a favore di terzo.

Analizzando la disciplina normativa è pacifico asserire che nel contratto di accollo è possibile riscontrare la presenza di tutti gli elementi propri di una stipulazione a favore di terzo: in primo luogo vi è una pattuizione avente ad oggetto tra debitore-stipulante ed accollante-promittente l'assunzione del debito che, a prescindere dall'adesione del terzo creditore accollatario, ha una sua causa ed è sorretta da un interesse delle parti che giustifica l'instaurazione del vincolo contrattuale in assenza di un'adesione.

In secondo luogo il codice civile all'art. 1273 conferisce al soggetto estraneo ma indirettamente beneficiato dal contratto di accollo di aderire alla pattuizione per rendere irrevocabili nei suoi confronti gli effetti della stipulazione profittando dell'estensione della responsabilità debitoria all'accollante, in piena coincidenza con quanto prescritto dall'art. $1411 \mathrm{cod}$. civ.

Tale inquadramento risolve in modo definitivo il problema della causa variabile del negozio in quanto per la validità della stipulazione di una pattuizione a favore del terzo è indispensabile dal punto di vista funzionale l'esistenza del solo interesse (espresso) dello stipulante alla conclusione dell'accollo che in tal caso è identificato nella causa solvendi comune ai negozi di assunzione del debito altrui.

Effetto di tale inquadramento è l'obbligo per il terzo accollante di adempiere in luogo dell'originario debitore anche prima dell'adesione del creditore alla stipulazione, poiché quest'ultima ha solo l'effetto di rendere irrevocabile la pattuizione e non anche di perfezionare il contratto, non va inoltre sottaciuto che i contraenti possono concordemente decidere di escludere la facoltà del creditore accollatario di aderire alla stipulazione, in tal caso l'accollo cd. "interno" avrà effetti favorevoli per il terzo solo in modo riflesso.

\section{Disciplina}

L'accollo è il negozio di assunzione del debito altrui che presenta una moltitudine di varianti giuridiche con notevoli riflessi sulla disciplina della pattuizione.

In primo luogo va rimarcata la distinzione tra accollo cumulativo ed accollo liberatorio fondata sulla "sorte" del debitore originario.

La prima fattispecie, che costituisce il prototipo legale di accollo, determina la sola aggiunta del terzo accollante al debitore accollato modificando soggettivamente l'obbligazione con l'allargamento della platea dei soggetti tenuti all'adempimento.

A differenza però dell'espromissione e della delegazione cumulativa il legame solidale che si viene a creare tra il vecchio ed il nuovo debitore ha una portata diversa a seconda della natura del contratto di accollo e del comportamento attivo del creditore beneficiario.

Qualora infatti l'accollo sia meramente interno o, seppur costruito come contratto a favore di terzo, non vi sia ancora stata l'adesione del beneficiario, avremo un'obbligazione solidale in senso stretto che impedisce la regressione della posizione dell'accollato e priva il debitore originario del beneficium ordinis.

La sussidiarietà dell'obbligazione solidale generata dall'accollo potrà avere effetto solo quando

il contratto a favore di terzo avrà spiegato i suoi effetti in modo pieno e definitivo attraverso l'adesione del creditore.

L'atto con cui l'accollatario profitta della stipulazione in suo favore ha pertanto il duplice effetto di rendere da un lato irrevocabile la stipulazione in suo favore e dall'altro applicabile il principio generale sancito in materia di negozi di assunzione del debito altrui dall'art. 1268 cod. civ.

Con una tale manifestazione di volontà il creditore accollatario rende manifesta la conoscenza 
dell'accordo di modificazione della posizione soggettiva del rapporto obbligatorio accettando le conseguenze giuridiche che la legge fa scaturire dall'adesione del creditore ai negozi di assunzione del debito da parte di un terzo.

Per ciò che invece concerne l'accollo liberatorio, anche in tal caso il consenso del creditore beneficiario è indispensabile alla estromissione del vecchio debitore dal rapporto obbligatorio, tuttavia la liberazione può essere il frutto di una libera scelta del creditore che svincola l'accollato a mezzo di un atto unilaterale recettizio oppure può consistere in una "controprestazione" cui è tenuto nei confronti del debitore per effetto della stipulazione.

In tale ultimo caso la liberazione del debitore costituisce una condizione sospensiva dell'esercizio del potere di adesione al contratto in suo favore; tale tecnica contrattuale non determina l'inefficacia dell'accollo ma ne comprime i soli effetti esterni. L'accollo rimane interno fintantoché il creditore non aderisce liberando così il vecchio debitore. È chiaro che anche in tal caso vi è un vantaggio per il creditore che è quello di poter decidere o di fruire della responsabilità di un soggetto più solvibile dell'antico debitore, al prezzo di liberare quest'ultimo, o di lasciare vincolato il proprio debitore all'adempimento riservandosi in un momento più favorevole di aderire alla stipulazione e correre però il rischio di subire l'esercizio del potere di revoca da parte dello stipulante accollato.

La disciplina normativa non lascia il creditore privo di tutela a seguito della liberazione del debitore antico, infatti l'art. 1274 cod. civ. prevede per il caso di liberazione "vincolata" all'adesione all'accollo l'automatica responsabilità del vecchio debitore in caso di insolvenza preesistente all'adesione, dando inoltre la possibilità al creditore di riservarsi in modo espresso il potere di agire contro il debitore liberato nel caso in cui l'insolvenza dell'accollante sia sopravvenuta alla stipulazione e ciò anche in caso di liberazione del debitore per atto unilaterale. È bene ribadire che in tali casi il soggetto liberato risponde in garanzia e non a titolo di debitore pertanto sarà necessario per il creditore insoddisfatto dare dimostrazione di aver escusso infruttuosamente il debitore sopravvenuto.

La liberazione produce inoltre l'effetto previsto dall'art. 1275 cod. civ. di estinguere le garanzie annesse al credito non espressamente lasciate in vita dai garanti così come stabilito per l'espromissione e la delegazione liberatoria. L'effetto "purgativo" delle garanzie però non copre quelle prestate dal debitore originario ogni qual volta che l'accollo viene meno retroattivamente per effetto di una causa di invalidità, in tal caso infatti l'obbligazione del debitore originario rivive insieme alle garanzie personali dallo stesso prestate.

Dall'interpretazione giuridica della norma può derivarsi che a differenza che per il caso di insolvenza preesistente, in occasione di una causa di invalidità dell'accollo, la riviviscenza dell'originaria obbligazione è "piena" ed è a titolo di responsabilità. In pratica il debitore originario è come se fosse rimasto sempre il debitore principale in quanto l'invalidità ha effetti retroattivi e salva i soli diritti dei terzi in buona fede, che nel caso di specie sono identificabili nei soggetti garanti originari del debito diversi dall'obbligato.

Il regime delle eccezioni opponibili dal terzo accollante al creditore accollatario scaturenti dal rapporto di provvista è disciplinato in modo espresso dall'art. 1273 cod. civ. in base al quale il nuovo debitore impegnandosi ad adempiere nei limiti del contratto di accollo stipulato può eccepire al terzo beneficiario i vizi e le situazioni patologiche di natura oggettiva concernenti il contratto di accollo, rimanendo estranee alla negoziazione le sole eccezioni strettamente personali. 
Le eccezioni fondate sul rapporto di valuta sono invece opponibili per relationem alla disciplina prevista dall'art. 1272 cod. civ. il quale sancendo che con il negozio di assunzione del debito altrui il terzo assume il medesimo debito l'obbligato passivo originario determina la possibilità per l'accollante di far valere ogni eccezione di carattere reale caratterizzante il rapporto di valuta. Sono ovviamente escluse anche in tal caso le eccezioni fondate su presupposti soggettivamente legati alle parti del rapporto obbligatorio (difetto di capacità, cause di annullamento relative, ecc..).

Dal regime delle eccezioni può concludersi che il contratto di accollo, visto anche il suo inquadramento sub specie contratto a favore di terzo, è doppiamente titolato, essendo legato al rapporto di provvista ed a quello di valuta.

In base al principio di autonomia contrattuale che governa il nostro sistema giuridico, è ben possibile che accollante ed accollato stipulino un negozio di assunzione del debito con soli effetti interni. In tal caso la disciplina dell'accollo è applicabile all'accordo delle parti solo per relationem ed in riferimento ai rapporti debitore-terzo accollante, le parti infatti sono libere di escludere la possibilità che l'assunzione del debito abbia effetti esterni e coinvolga il creditore che può addirittura essere ignaro di una pattuizione modificativa del rapporto obbligatorio.

L'accollo interno è pertanto un contratto atipico che produce effetti solo relativamente al debitore ed al terzo accollante ed ha la funzione di trasferire tra le parti il peso economico del debito senza creare una modificazione soggettiva od un vincolo di solidarietà passiva di cui il creditore può profittare. Pertanto unico debitore escutibile dal creditore sarà l'accollato che in virtù del contratto di accollo interno potrà agire in rivalsa contro l'accollante in adempimento del patto di assunzione del debito pagato.

È necessaria però una distinzione tra accollo originariamente interno che consiste in un contratto atipico di assunzione del debito altrui stipulato tra debitore e terzo con espressa esclusione del potere del creditore di aderire alla stipulazione ed accollo interno derivante dal rifiuto del creditore di profittare della stipulazione in suo favore, in tal caso la mera rilevanza inter partes (accollante-accollato) è il frutto dell'effetto prodotto dal rifiuto del terzo ex art. 1411 cod. civ. pertanto il contratto originariamente esterno si converte in accollo interno in ossequio al principio di conservazione del contratto.

\section{DELEGAZIONE, ESPROMISSIONE, ACCOLLO: DIFFERENZE, FIGURE AFFINI ED APPLICAZIONI PRATICHE}

Delegazione, espromissione ed accollo sono istituti caratterizzati dalla comune funzione di determinare l'assunzione del debito altrui, differendo tra loro per struttura e natura giuridica, vista la rilevante differenza sul piano della disciplina ed in particolare delle eccezioni esperibili dai soggetti coinvolti, un'operazione di esatta qualificazione risulta essere indispensabile ogni qual volta l'operatore giuridico è alle prese con un negozio di assunzione del debito altrui.

Partendo dalla delegazione è necessario sottolineare la distinzione intercorrente sul piano strutturale con l'espromissione. Nella prima vi è una vera e propria operazione giuridica caratterizzata dalla presenza di tre atti negoziali causalmente autonomi ma collegati, nella seconda invece il momento negoziale è unico.

Entrambe le figure sono accomunate dalla marginalità del consenso del debitore rispetto al perfezionamento del negozio di assunzione del debito.

In verità l'espromissione intesa come accordo tra creditore e terzo è assimilabile ad uno degli elementi del procedimento delegatorio: il cd. negozio di assunzione del debito; tuttavia questo 
atto negoziale seppur esente nella sua genesi dalla necessità di una manifestazione di volontà del debitore-delegante non può dirsi caratterizzato dalla spontaneità tipica dell'espromissione. L'assunzione del debito da parte del delegato infatti seppur perfetta per il solo consenso prestato dal creditore non può ritenersi giustificata da un atto di spontaneità ma trova fondamento in un ordine del delegante che ha notevoli ripercussioni sul piano della disciplina delle eccezioni opponibili.

La struttura trilatera della delegazione distingue la stessa anche dal contratto di accollo in cui il coinvolgimento del terzo creditore è solo eventuale.

Le parti del contratto di assunzione del debito sono diverse: nella delegazione il terzo delegato assume l'obbligo verso il creditore mentre nell'accollo quest'ultimo ha solo la facoltà (ove riconosciutagli) di profittare del contratto di assunzione del debito che il terzo accollante conclude con il debitore originario; da tale strutturazione deriva un'ulteriore e determinante differenza tra gli istituti concernente il "tipo" di solidarietà che caratterizza il lato passivo. Nella delegazione la solidarietà assume carattere sussidiario e fa sorgere in capo al debitore delegante il beneficium ordinis, nell'accollo invece tale effetto è prodotto solo nell'eventualità di un'accettazione della stipulazione da parte del creditore beneficiario, fino a quel momento la solidarietà passiva è "pura" e non comporta alcuna degradazione della responsabilità del debitore accollato.

Le differenze intercorrenti tra espromissione ed accollo sono invece di carattere soggettivo, entrambe le figure infatti hanno natura contrattuale e determinano l'assunzione del debito altrui; l'espromissione è tuttavia un contratto al quale è estranea la posizione del debitore in quanto si fonda su un accordo raggiunto tra terzo espromittente e creditore, il debitore originario può addirittura essere all'oscuro dell'assunzione del debito che avviene per effetto di una spontanea iniziativa del terzo. Nell'accollo invece ad essere estraneo alla contrattazione è il creditore che si avvantaggia della stipulazione solo se le parti non escludono l'estensione degli effetti favorevoli alla sua sfera giuridica; l'assunzione del debito è pertanto validamente conclusa solo con il consenso di debitore e terzo e può non avere alcun effetto verso il creditore. Ovviamente la più rilevante ripercussione della diversità strutturale soggettiva è data dal regime delle eccezioni che fanno dell'espromissione un contratto parzialmente astratto, svincolato dal rapporto sussistente tra debitore ed espromittente, a differenza dell'accollo che è doppiamente titolato e subisce gli effetti di entrambe i rapporti di base.

Analizzate le distinzioni strutturali sussistenti tra $i$ tre istituti è ora indispensabile sottolineare le affinità che ognuno di essi presenta con negozi ed istituti giuridici funzionalmente differenti. Una prima distinzione va fatta tra delegatio promittendi e fideiussione.

L'affinità tra le due figure nasce dal comune scopo degli istituti di estendere la responsabilità dell'adempimento a più soggetti, va però precisato che mentre la responsabilità del terzo derivante dalla delegazione è a titolo di debito, quella determinata dalla fideiussione è a titolo di garanzia pertanto il fideiussore è un garante che è chiamato a rispondere innanzitutto in tale veste e quindi solo in caso di inadempimento del debitore che resta l'unico obbligato principale, ed in secondo luogo va ricordato che il peso economico è pienamente a carico del soggetto garantito che sarà tenuto alla restituzione di quanto pagato dal garante che può surrogarsi nei diritti del creditore soddisfatto.

La differenza del titolo di responsabilità si riflette altresì sulla sorte del rapporto: mentre la delegazione non viene meno nel caso di vizi che inficiano il rapporto di valuta, a meno che non 
vi sia un espresso richiamo fatto dalle parti ex art. 1271 cod. civ., la fideiussione perde efficacia ogni volta che l'obbligazione garantita si estingue, in quanto ad essa accessoria.

Stesse differenze possono essere richiamate per distinguere la fideiussione dall'espromissione. Affinità relativamente agli effetti satisfattivi dell'obbligazione sono rinvenibili anche tra delegazione di pagamento ed adempimento del terzo. In entrambe i casi infatti il pagamento effettuato dal terzo estingue il rapporto di valuta, tuttavia la delegazione nasce da un atto di incarico del delegato al delegante, configurandosi come atto dovuto in adempimento di un rapporto di mandato, e spesso comporta uno spostamento del peso economico sul delegato che non può agire in regresso se non qualora il debitore abbia giovato di un indebito oggettivo. L'adempimento del terzo è invece caratterizzato dalla spontaneità del pagamento e permette al terzo adempiente di agire in regresso o di richiedere al creditore la surrogazione nel credito pagato.

La distinzione tra adempimento del terzo ed espromissione è netta in quanto con il primo negozio il terzo assume il debito altrui senza soddisfare il creditore, nel secondo caso invece l'obbligazione si estingue ed al terzo spetta solo l'eventuale surrogazione nei diritti del creditore o l'azione di regresso verso il debitore liberato.

Se è vero che gli istituti esaminati hanno struttura e funzione tipica, in quanto servono, come detto ad estendere la responsabilità del debitore ad un terzo assuntore, è altrettanto vero che in base al principio di autonomia contrattuale l'impiego di tali figure negoziali è spesso plasmato per soddisfare interessi diversi da quelli protetti dal codice. L'espromissione o la delegazione di debito risultano essere strumenti negoziali utili all'elargizione di una liberalità o al pagamento di altre obbligazioni o alla creazione ex novo di una ragione di credito onerosa, in altri casi ancora è la stessa legge a richiedere l'impiego di un atto negoziale di assunzione del debito per scopi diversi dalla mera modificazione della posizione soggettiva.

Il favore per l'uso di tali negozi è legato all'evolversi della contrattazione negoziale e delle esigenze sociali che richiedono strumenti nuovi di circolazione della ricchezza.

In tale ottica i negozi di assunzione del debito sono congeniali ad assicurare ai creditori coinvolti nelle operazioni di circolazione del credito la possibilità di permettere il mutamento del soggetto passivo mantenendo in vita la responsabilità del vecchio soggetto obbligato ed al debitore di poter gestire la propria situazione finanziaria in modo più agile senza dover ricorrere ad operazioni contrattuali lunghe e dispendiose permettendogli di trarre benefici economici disponendo di rapporti già in essere [si faccia l'esempio di un'imprenditore-debitore che, in assenza di liquidità, ricorre alla delegazione di pagamento per soddisfare un proprio fornitore, incaricando un suo cliente di pagare il saldo della propria fattura direttamente al creditore. In tal caso non vi è la necessità dell'imprenditore di dover ricorrere all'apertura di una linea di credito verso il fornitore per soddisfare le proprie obbligazioni, ed al contempo gioverà dell'immediato incasso di un saldo in grado di generare un apporto contabile doppiamente positivo (incasso di un credito e pagamento di un debito). Dall'altra parte il fornitore soddisfatto non correrà il rischio dell'insolvenza dell'imprenditore in quanto garantito dal patrimonio di due soggetti senza dover dimostrare tra l'altro in nomen verum del credito per richiedere il pagamento al delegato; a sua volta il cliente delegato potrà chiedere al delegatario una dilazione di pagamento o potrà ottenere dall'imprenditore uno sconto sul saldo finale in cambio dell'immediato adempimento verso il fornitore]. 
Dal punto di vista pratico uno dei negozi che meglio si presta alla creazione di una ragione di credito o all'elargizione di una liberalità indiretta è l'espromissione. Per quanto detto infatti l'assunzione del debito da parte dell'espromittente non è vincolata ad un incarico del debitore in quanto è generata da una volontà spontanea del terzo.

È proprio l'estraneità alla produzione dell'effetto tipico dell'espromissione del rapporto espromittente-espromesso a fare della stessa un duttile strumento negoziale.

È infatti possibile "sfruttare" la causalità dell'espromissione per giustificare un atto donativo dall'espromittente al debitore senza dover soggiacere agli obblighi formali previsti dalla legge per la donazione, oppure costituirsi una ragione di credito a titolo di mutuo/finanziamento senza dover pagare immediatamente il creditore espromissario (il terzo si "assume" il debito, l'espromissione non è satisfattiva). Si faccia l'esempio del terzo espromittente che assumendosi l'obbligo del pagamento verso il creditore principale ottiene la liberazione dell'originario debitore al solo fine di riottenere da quest'ultimo la restituzione del tandundem a titolo di mutuo; si consideri inoltre l'ipotesi del terzo espromittente creditore dell'espromesso che non agisce nell'interesse di liberare il debitore ma assume il debito altrui per compensare (volontariamente) il proprio credito (magari poco garantito o molto dilazionato) al solo fine di poter agire in restituzione verso l'antico debitore espromesso ed ottenere immediatamente la somma pagata (ovviamente in tal caso l'espromittente diventato creditore dell'espromesso può ottenere solo ciò che il debitore doveva al creditore originario in base al rapporto di valuta, il vantaggio può consistere nell'ottenimento di un pagamento immediato a fronte di quello dilazionato opposto in compensazione o addirittura giovare di un debitore più solvibile del primo).

L'espromissione costituisce inoltre un utile strumento di risoluzione della crisi d'impresa sia nel caso in cui sull'imprenditore penda una procedura concorsuale sia qualora il medesimo è in una situazione di impossibilità di adempiere con regolarità alle proprie obbligazioni. Ovviamente ricorrere all'espromissione significa adottare uno strumento atipico con effetti diversi da quelli previsti dal codice della crisi d'impresa come la cessione dei beni ai creditori o l'assunzione del concordato fallimentare/preventivo. Nel caso di espromissione infatti non si verifica un automatico subingresso dell'espromittente nel possesso dei beni dell'imprenditore, che potrà eventualmente essere perfezionato solo successivamente al pagamento dei debiti in via di datio in solutum per il soddisfacimento dei creditori della massa.

È chiaro che per potersi parlare di espromissione il terzo deve intervenire spontaneamente e nel caso di intervento manente fallimento è necessario il consenso degli organi della procedura oltre che dei creditori, qualora invece l'intervento del terzo assuntore derivi dall'incarico dell'imprenditore l'assunzione sarà delegazione e non espromissione.

Per quanto detto in materia di accollo, con particolare riferimento alla sua natura di contratto a favore di terzo la duttilità del negozio è in re ipsa. Nella prassi negoziale infatti è frequentissimo il ricorso all'assunzione del debito altrui con effetto interno alle parti in particolare nella contrattazione immobiliare ove spesso i beni oggetto di trasferimento sono gravati da ipoteche iscritte a garanzia dell'adempimento di contratti di mutuo fondiario. In tali casi l'accollo costituisce una sostanziale modalità di pagamento del prezzo del bene, anche se sul piano formale rimane un contratto di assunzione del debito altrui (non vi sarà l'obbligo di inserire l'accollo nella dichiarazione ex art. co. 36 d. L. 223/2006 non essendovi un immediato trasferimento di danaro).

Ciò che però rileva in materia di accollo è il suo impiego "legale", in non poche occasioni infatti è proprio il dettato normativo a prevedere, in conseguenza di un atto di trasferimento o 
costituzione di una situazione giuridica soggettiva, la nascita di un contratto di accollo per i soggetti coinvolti.

Sono ipotesi di accollo legale cumulativo quelle stabilite negli artt.: 2112 cod. civ. concernente i crediti del lavoratore anche non contabilizzati che per effetto della cessione d'azienda devono essere pagati solidalmente da imprenditore cedente e cessionario; 1546 cod. civ. relativamente all'accollo dei debiti ereditari per l'acquirente in caso di vendita di eredità da parte del delato; 2269 cod. civ. che prevede l'obbligo per il socio cessionario di quota di società di persona di pagare in solido con il socio uscente i debiti sociali esistenti al momento dell'acquisto; $2356 \mathrm{e}$ 2472 cod. civ. in materia di trasferimento di partecipazioni di società di capitali che prevedono l'obbligo in solido per l'acquirente per i versamenti dei centesimi ancora dovuti dal cedente.

È invece liberatorio l'accollo legale sancito dall'art. 2558 cod. civ. in caso di trasferimento d'azienda quando è disposta la liberazione del cedente verso i potenziali creditori di contratti stipulati dall'imprenditore per l'esercizio dell'impressa che comportano per la loro natura l'automatico subingresso del nuovo imprenditore.

Vi sono poi rare ipotesi di accollo legale "interno" in cui la legge dispone il solo trasferimento del peso economico del debito in capo al terzo lasciando immutate le pretese dei creditori verso l'originario debitore, come nel caso di gestione d'affari altrui non rappresentativa ex art. 2031 cod. civ. che obbliga il soggetto interessato all'utile gestione del suo patrimonio a tenere indenne dalle obbligazioni assunte il terzo che abbia spontaneamente curato i suoi interessi.

Tra le varie utilizzazioni pratiche dei negozi di assunzione del debito altrui va menzionata la possibilità di porre in essere, con efficacia diretta, una delegazione mortis causa mediante una disposizione testamentaria.

Per la struttura trilatera che la caratterizza la delegazione è infatti l'unico negozio di assunzione del debito altrui che può essere impiegato dal de cuius con efficacia diretta, ciò in quanto è precluso al testatore il potere di creare con il testamento, che è atto unilaterale mortis causa, situazioni giuridiche a carattere contrattuale che oltre a richiedere il consenso dell'altro contraente, corrono il rischio di essere annullate per violazione del divieto di patto successorio (dispositivo); va inoltre ricordato che il de cuius non può in alcun modo alterare la par condicio creditorum creando con il testamento, cause legittime di prelazione o garanzie con efficacia reale in grado di pregiudicare i creditori ereditari.

Pertanto l'unico modo di determinare in modo diretto e con effetto reale l'assunzione del debito altrui mortis causa è quello di ricorrere all'incarico delegatorio. In tal caso però devono verificarsi diverse condizioni: in primo luogo il soggetto delegato deve essere debitore del de cuius e deve essere istituito erede (la prima condizione è richiesta per evitare che la delegazione assuma il carattere di una proposta contrattuale, mentre la seconda è effetto del principio generale per cui il testamento non può onerare un soggetto estraneo alla successione), in secondo luogo il debito che l'erede-debitore è incaricato di assumere deve appartenere al patrimonio del de cuius. Così facendo il debitore con atto di delegazione avente efficacia diretta (seguendo la teoria dello iussum unilaterale) otterrà per effetto dell'accettazione dell'eredità l'assunzione automatica del debito senza la necessità di accettazione da parte del delegato.

A suffragio di tale impostazione è possibile richiamare sia l'art. $1315 \mathrm{cod}$. civ. che prevede la possibilità per il testatore di "incaricare" uno dei coeredi all'esecuzione di una disposizione testamentaria al di fuori dello stato di comunione (in deroga al principio "debita hereditaria ipso iure dividuntur"), sia dalle stesse norme dettate in tema di delegazione ove è stabilito che il 
delegato può eseguire l'incarico anche successivamente alla morte del testatore, presupponendo dunque che la delega possa essere contenuta in un testamento in quanto disposizione a carattere patrimoniale. 


\section{BIBLIOGRAFIA ESSENZIALE}

- L. Genghini - R. Apicella, "Le Obbligazioni”, MANUALI NOTARILI vol. VI, 2015, CEDAM, Padova;

- M. Allara, "Delle obbligazioni” Lezioni raccolte da Deiana, Torino, 1939;

- M. Andreoli, "La delegazione”, in Studi di diritto privato, CEDAM, Padova, 1937;

- L. Barassi, “La teoria generale delle obbligazioni”, vol.I, GIUFFRE', Milano, 1964;

- C. Barnini, “Obbligazione”, in Enciclopedia Giuridica, XXI, TRECCANI, Roma, 1990;

- E. Betti, “Teoria generale delle obbligazioni”, vol. I, GIUFFRE', Milano, 1953;

- C. M. Bianca, "Diritto Civile, L'obbligazione”, vol. IV, GIUFFRE', Milano, 1993;

- L. Bigliazzi Geri - U. Breccia - F.D. Busnelli - U. Natoli, "Diritto civile, obbligazioni e contratti", vol. III, UTET, Torino, 1989;

- W. Bigiavi, “La delegazione”, CEDAM, Padova, 1940;

- B. Biondi, "La causa della espromissione" in Foro italiano, p. 1335, I, 1956,

- E. Briganti - D. Valentino, "Le vicende delle obbligazioni. La circolazione del debito e del credito" in Trattato di diritto civile del Consiglio Nazionale del Notariato, ESI, Napoli, 2007;

- F.D. Busnelli, "l'obbligazione soggettivamente complessa. Profili sistematici”, GIUFFRE', Milano, 1974;

- G. Campobasso, “accollo”, in Enciclopedia Giuridica, I, TRECCANI, Roma, 1988;

- C. A. Cannata, "l'adempimento delle obbligazioni”, in Trattato Rescigno, 9, UTET, Torino, 1984;

- C. A. Cannata, "le obbligazioni in generale", in Trattato Rescigno, 9, UTET, Torino, 1984

- A. Chianale, “l’obbligazione”, in Digesto Civile, vol. XII, UTET, Torino, 1995;

- R. Cicala, "Espromissione", in Enciclopedia giuridica, XIII, TRECCANI, Roma, 1987;

- R. Cicala, “Accollo”, in Enciclopedia del diritto, I, GIUFFRE', Milano, 1958;

- A. Di Majo, "Delle obbligazioni in generale" in Commentario Scialoja e Branca, Bologna-Roma, sub. Artt. 1173-1176, 1988;

- F. Ferrara jr. - F. Corsi, “Gli imprenditori e le società", GIUFFRE', Milano, 2006;

- F. Galgano, "Diritto civile e commerciale", vol. II, CEDAM, Padova, 1999;

- F. Galgano, "Le obbligazioni in generale", CEDAM, Padova, 2011; 
- F. Gazzoni, "Manuale di diritto privato”, ESI, Napoli, 2003;

- G. Giacobbe - D. Giacobbe, "Delegazione, espromissione, accollo (art. 1268-1273)", in Commentario Scialoja e Branca, Bologna-Roma, 1992;

- C. Lazzara, "Accollo e lberazione del debitore originario", in Rivista trimestrale di diritto e procedura civile, GIUFFRE', Milano, 1957;

- F. Messineo, "Manale di diritto civile e commerciale”, vol.I, GIUFFRE', Milano, 2009;

- F. Santoro-Passarelli, “Dottrine generali di diritto civile”, ESI, Napoli, 2002; 